\title{
Looking to the Future: A Fermilab Viewpoint
}

\author{
H. E. Montgomery ${ }^{\mathrm{a}}$ \\ ${ }^{a}$ Fermi National Accelerator Laboratory, \\ P.O. Box 500, Batavia, Illinois 60510, U.S.A.
}

This is a short paper summarising a presentation of the evolution of the Fermilab program for the next five to ten years. Emphasis is given to the Fermilab accelerator complex, but external collaboration is emphasised.

\section{Introduction}

An informal public discussion was held near the end of the program of presentations at the High Intensity Frontier Workshop on Elba in the spring of 2005. This was close to the point (July 1, 2005) at which Fermilab changed its Director. At the time of the discussion, the incoming Director, Piermaria Oddone, had made a presentation [1] giving his vision for the laboratory. While outlining the current program and looking toward potential directions for collaboration, this presentation and paper leans heavily on Oddone's presentation.

\section{Current Program}

The future planning at Fermilab is founded on the current accelerator-based physics program. This program is dominated by the Tevatron Collider experiments which enjoy the highest energies available in the laboratory and will continue to do so until the Large Hadron Collider (LHC) comes online at CERN later in this decade. At present approximately one $f b^{-1}$ of integrated luminosity has been delivered to each experiment. Peak instantaneous luminosities of greater than $10^{32} \mathrm{~cm}^{-2} \cdot \mathrm{sec}^{-1}$ are common and prospects for yet higher luminosity are bright.

The neutrino program now has several components. The MiniBooNE experiment is searching for neutrino oscillations corresponding to the indications observed by the LSND experiment at Los Alamos a few years ago. This experiment has received approximately $6 \times 10^{20}$ protons on target from the Booster Accelerator at $8 \mathrm{GeV}$; we are eagerly looking forward to the results. During the past year the Neutrino at the Main Injector (NuMI) beamline was completed, commissioned, and is now operating. The MINOS experiment, a long baseline neutrino oscillation experiment using that beam line, hopes to receive a good fraction of $10^{20}$ protons on target by years end. We hope soon to mount the MINER $\nu$ A neutrino scattering experiment. To complement these immediate endeavours there has also been $\mathrm{R} \& \mathrm{D}$ on muon cooling related to future neutrino factories

Fermilab has been a major player in the construction of the interaction region magnets for the Large Hadron Collider (LHC), which is being constructed at CERN in Europe. This has been in collaboration with two other US laboratories and with the KEK laboratory in Japan. Fermilab is the host laboratory for US-CMS, the collaboration which is building the U.S. contributions to the Compact Muon Solenoid (CMS) experiment. Fermilab physicists have enjoyed leadership rôles in the construction project and the computing and software program. They are now taking the lead in the maintenance and operation. Recently an LHC Physics Center (LPC) was initiated; it is hoped that this will enable US scientists to enhance their places in the analysis phases of the experiment and that CMS will see increased activity in this vital area.

The Laboratory is host to a suite of astroparticle physics experiments which include the Sloan Digital Sky Survey, The Pierre Auger cosmic ray observatory, and the Cryogenic Dark Matter Search experiment in the Soudan mine. Each of 
these is a world leader and on this base we are developing a new Dark Energy Survey experiment.

The experimental programs of the laboratory are supported by two excellent theoretical groups in particle and particle astrophysics.

Nevertheless, the name of the laboratory contains the word accelerator. It is in accelerator $\mathrm{R} \& \mathrm{D}$ for the future, and for the experiments to go with those accelerators, where we have seen the largest number of contributions from the laboratory to this workshop. These are perhaps the most important things we bring to the table.

\section{New Initiatives}

The overarching goal for the laboratory will be to enable the most powerful attack possible on the fundamental science questions by providing world class facilities. These facilities will be integrated in a global network. It is necessary to develop science and technology for particle and astroparticle physics research.

Specific goals are to make vital contributions during the next decade to developing a powerful new tool for discovery, the International Linear Collider. This will be achieved while maintaining the foremost neutrino program through the development of the $\mathrm{NO} \nu \mathrm{A}$ experiment and enhancements to the Fermilab proton source.

To contribute 2 to the International Linear Collider, it is now critical to establish, within the U.S., world class expertise in super-conducting radio-frequency technology. There are facilities at Fermilab, buildings which exist, which are being turned to this goal. The work will be integrated into the global effort. That effort has an immediate aim to generate a conceptual design for the machine by the end of 2006. At that juncture, it is hoped that there will be sufficient information to inform the choice of paths forward. If the design looks as though it fits to both the needs of the science and that of the governments then it will be important to complete the development work to enable a decision to construct or not as early as possible. If there are major issues, it may be necessary to embark on a longer development program.

On the neutrino front, Fermilab is currently operating a neutrino program which enjoys the most powerful proton beam available to that end in the world. By year end we can see operation for MINOS the long baseline oscillation experiment with a beam power of $200 \mathrm{KW}$. Upgrades could get that to $450 \mathrm{KW}$ by 2008 . If the Tevatron Collider program ends in 2009, that would make in excess of $600 \mathrm{KW}$ available. Imaginative ideas suggest potential in the $1 \mathrm{MW}$ range at 120 $\mathrm{GeV}$.

However, the existing complex is old and the path beyond $1 \mathrm{MW}$ with that complex is daunting. A new source, dubbed the Fermilab Proton Driver [3] (FPD), would alleviate most of these issues and provide a platform for high intensity proton source operation over a range of energies for physics. It is currently envisaged that this FPD would be an $8 \mathrm{GeV}$ super-conducting linear accelerator. At low energy, less than $1 \mathrm{GeV}$, its concepts are borrowed from the Spallation Neutron Source (SNS) being built at Oak Ridge National Laboratory. At high energies it would have many similarities to the Tesla design for the linear collider. There appears to be considerable synergy with the work needed for the ILC.

The currently operating MINOS experiment is well matched by the current NuMI beam. That beam with the enhancements outlined above over the next few years could support the search for $\nu_{\mu}$ to $\nu_{e}$ oscillations with a long baseline. This is a key to the next step in understanding the neutrino flavor physics. The experiment has been named $\mathrm{NO} \nu \mathrm{A} 4$. Its current design envisages a mass of about 20-30 ktonnes of liquid scintillator and polyvinyl-chloride tube container. The very long baseline would enable a unique sensitivity to the neutrino mass hierarchy. Thus, $\mathrm{NO} \nu \mathrm{A}$ is seen as the second step, after MINOS in a flexible approach to the neutrino physics puzzles. Since the figure of merit involves also the detector sensitivity we also foresee starting a program of $R \& D$ into the use of liquid argon technology [5] which might have potential for the phase after $\mathrm{NO} \nu \mathrm{A}$.

The physics that one can achieve with a proton driver is extensive [6; ; it could potentially include high intensity muon, pion and kaon experiments as demanded by the science. In fact, when we consider the potential of intense proton sources 
for visionary programs such as neutrino factories, the Fermilab Proton Driver could provide an important and broad launch platform.

\section{Opportunities for Collaboration}

No single laboratory is going to do all, or even half, of particle physics alone and Fermilab recognises that collaboration is essential. This ranges from the International Linear Collider where Fermilab expects to collaborate on superconducting technology, in the ILC machine and on the preparations for the experiments.

The enhancement of the proton source and the R\&D towards a Proton Driver already takes from the designs of others and even at this meeting some new opportunities have opened up. It was emphasised above that a major component of Fermilab research, both accelerators and experiments is associated with the LHC program at CERN. We are already involved in R\&D towards a luminosity upgrade for that machine and we expect this to continue. Rounding out our accelerator work we have an important but modest involvement in muon beam cooling and a participation in the MICE experiment.

On the experimental side, we have been delighted by the participation from all over the world in the Tevatron Collider program and, to a lesser extent, in the neutrino program. We would like to see Fermilab continue as one of the homes of the international particle physics community. To that end, many may be receiving invitations to join in the $\mathrm{NO} \nu \mathrm{A}$ initiative; we also expect that the liquid argon initiative will lead to collaboration.

Finally, it is clear that the limited resources have bitten into the kaon based flavor programs across the world. At Fermilab, the recent direction has been toward the measurement of the decay $K \rightarrow \pi \nu \bar{\nu}$ which appears to be the most readily accessible. Since it has not been possible to build such an experiment at Fermilab, we intend to conduct discussions as to whether a sensible U.S. participation in such an experiment at CERN could be mounted.

\section{Summary}

At Fermilab, we are enjoying an exciting present. There is a very healthy program currently operating with luminosity and intensities increasing regularly and physics results and publications appearing apace.

For the future we seek to be host to the International Linear Collider and to build on our neutrino program by improving our source and preparing the Fermilab Proton Driver.

To achieve this we must expand the extent of our collaboration with all.

\section{REFERENCES}

1. P. J. Oddone, presentation to National Academy Study "Elementary Particles in the 21st Century", May 16, 2005.

2. S. Mishra, Proton Driver-International Linear Collider Synergy, contribution to the H.I.F. Workshop, La Biodola, Elba, Italy, May 2005.

3. W. Foster, A Proton Driver at FNAL, contribution to the H.I.F. Workshop, La Biodola, Elba, Italy, May 2005.

4. R. Ray, The NO $\nu \mathrm{A}$ Experiment, contribution to the H.I.F. Workshop, La Biodola, Elba, Italy, May 2005.

5. A. Para, FLARE: Large Liquid Argon Detector for Oscillation Experiment at NuMI, contribution to the H.I.F. Workshop, La Biodola, Elba, Italy, May 2005.

6. S. Geer, Physics at the FNAL Proton Driver, contribution to the H.I.F. Workshop, La Biodola, Elba, Italy, May 2005; hep-ph/0507236. 